\title{
Corrigendum
}

\section{Corrigendum to "Improved Combinatorial Benders Decomposition for a Scheduling Problem with Unrelated Parallel Machines"}

\author{
Francisco Regis Abreu Gomes ${ }^{1}$ and Geraldo Robson Mateus ${ }^{2}$ \\ ${ }^{1}$ Graduate Program in Production Engineering, Federal University of Minas Gerais and Federal Institute of Education, \\ Science and Technology of Ceará, Belo Horizonte, MG, Brazil \\ ${ }^{2}$ Computer Science Department, Federal University of Minas Gerais, Belo Horizonte, MG, Brazil
}

Correspondence should be addressed to Francisco Regis Abreu Gomes; regisgomes@ifce.edu.br

Received 14 November 2017; Accepted 16 November 2017; Published 17 December 2017

Copyright (c) 2017 Francisco Regis Abreu Gomes and Geraldo Robson Mateus. This is an open access article distributed under the Creative Commons Attribution License, which permits unrestricted use, distribution, and reproduction in any medium, provided the original work is properly cited.

In the article titled "Improved Combinatorial Benders Decomposition for a Scheduling Problem with Unrelated Parallel Machines" [1], there were errors in Table 4, which should be corrected as follows. 
TABLE 4: Comparison of T\&B and ICBD relative to traditional and proposed convergence acceleration elements.

\begin{tabular}{|c|c|c|c|c|c|c|c|c|c|c|}
\hline \multirow{2}{*}{$n$} & \multirow{2}{*}{$m$} & \multicolumn{2}{|c|}{$\mathrm{T} \& \mathrm{~B}$} & \multicolumn{7}{|c|}{ ICBD } \\
\hline & & \# iter & \# cut & $\#$ ta & \# ws iter & \# iter & \# ws cut & \# cut & ws time & Normal time \\
\hline \multirow{4}{*}{20} & 2 & 3.73 & 7.47 & 0.30 & 4.27 & 1.47 & 12.93 & 4.13 & 0.74 & 0.33 \\
\hline & 3 & 4.53 & 13.60 & 0.60 & 5.20 & 1.40 & 24.10 & 5.60 & 1.13 & 0.75 \\
\hline & 4 & 5.17 & 20.67 & 0.53 & 5.00 & 1.30 & 36.40 & 6.53 & 2.33 & 1.93 \\
\hline & 5 & 5.37 & 26.83 & 0.47 & 4.80 & 1.13 & 44.67 & 6.33 & 5.22 & 6.44 \\
\hline \multirow{4}{*}{30} & 2 & 5.40 & 10.80 & 0.53 & 5.17 & 2.07 & 15.47 & 6.53 & 1.89 & 1.18 \\
\hline & 3 & 9.77 & 29.30 & 1.33 & 6.63 & 1.80 & 40.60 & 8.40 & 4.71 & 6.28 \\
\hline & 4 & 9.77 & 39.07 & 1.57 & 6.43 & 1.63 & 60.40 & 10.40 & 23.96 & 59.3 \\
\hline & 5 & 7.93 & 39.67 & 0.90 & 5.67 & 1.23 & 62.17 & 7.50 & 50.54 & 35.07 \\
\hline \multirow{4}{*}{40} & 2 & 4.73 & 9.47 & 0.47 & 4.77 & 2.00 & 14.87 & 6.87 & 3.09 & 1.84 \\
\hline & 3 & 8.50 & 25.50 & 1.63 & 6.47 & 1.43 & 39.50 & 5.70 & 8.34 & 7.75 \\
\hline & 4 & 12.23 & 48.93 & 1.77 & 7.87 & 1.33 & 76.13 & 7.20 & 65.99 & 45.53 \\
\hline & 5 & 9.17 & 45.83 & 1.97 & 6.97 & 1.47 & 89.83 & 10.50 & 374.79 & 369.05 \\
\hline \multirow{4}{*}{50} & 2 & 5.07 & 10.13 & 0.90 & 5.17 & 2.30 & 16.67 & 7.53 & 6.56 & 3.4 \\
\hline & 3 & 14.27 & 42.80 & 3.73 & 7.30 & 3.90 & 48.10 & 22.50 & 20.61 & 83.28 \\
\hline & 4 & 13.10 & 52.40 & 2.90 & 8.93 & 2.00 & 92.40 & 12.13 & 345.19 & 303.00 \\
\hline & 5 & 11.43 & 57.17 & 3.57 & 8.47 & 1.57 & 130.50 & 14.50 & 1083.98 & 947.89 \\
\hline \multirow{4}{*}{60} & 2 & 7.70 & 15.40 & 1.53 & 5.80 & 2.70 & 20.20 & 10.40 & 11.34 & 8.39 \\
\hline & 3 & 16.97 & 50.90 & 4.60 & 9.03 & 3.57 & 69.00 & 23.50 & 58.43 & 188.97 \\
\hline & 4 & 14.03 & 56.13 & 4.53 & 10.40 & 1.73 & 114.67 & 12.40 & 485.55 & 443.66 \\
\hline & 5 & 9.17 & 45.83 & 4.00 & 8.30 & 1.33 & 126.67 & 12.67 & 1309.28 & 1179.53 \\
\hline \multicolumn{2}{|c|}{ Average } & 8.90 & 32.40 & 1.89 & 6.63 & 1.87 & 56.76 & 10.07 & 193.18 & 184.68 \\
\hline
\end{tabular}

\section{References}

[1] F. R. A. Gomes and G. R. Mateus, "Improved combinatorial benders decomposition for a scheduling problem with unrelated parallel machines," Journal of Applied Mathematics, vol. 2017, Article ID 9452762, 10 pages, 2017. 


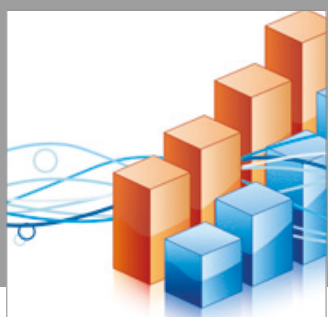

Advances in

Operations Research

vatersals

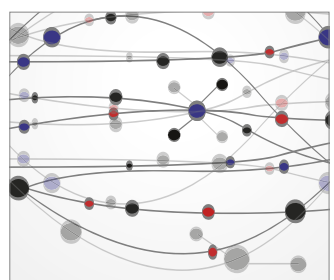

\section{The Scientific} World Journal
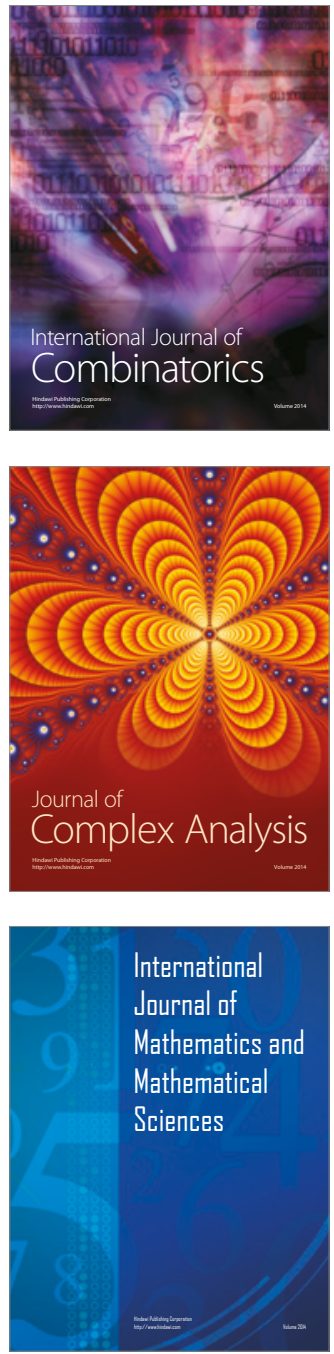
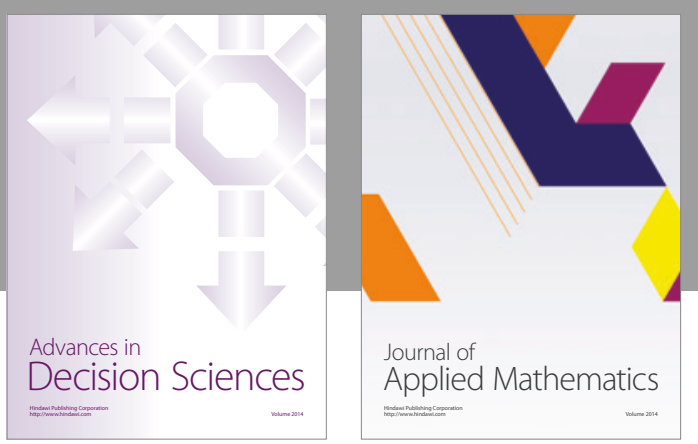

Algebra

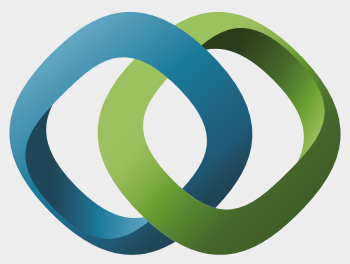

\section{Hindawi}

Submit your manuscripts at

https://www.hindawi.com
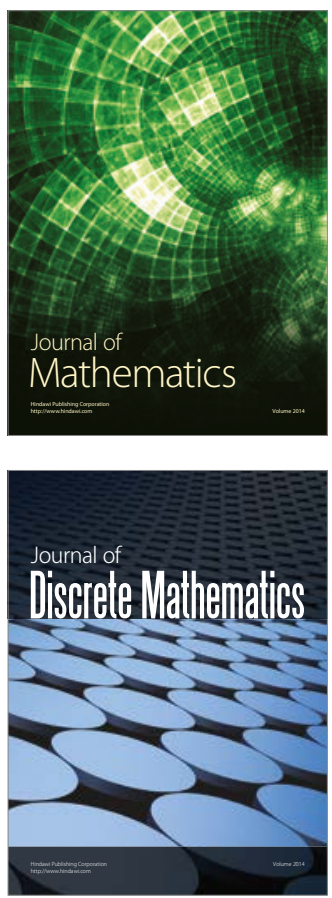

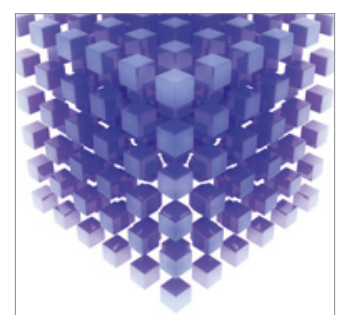

Mathematical Problems in Engineering
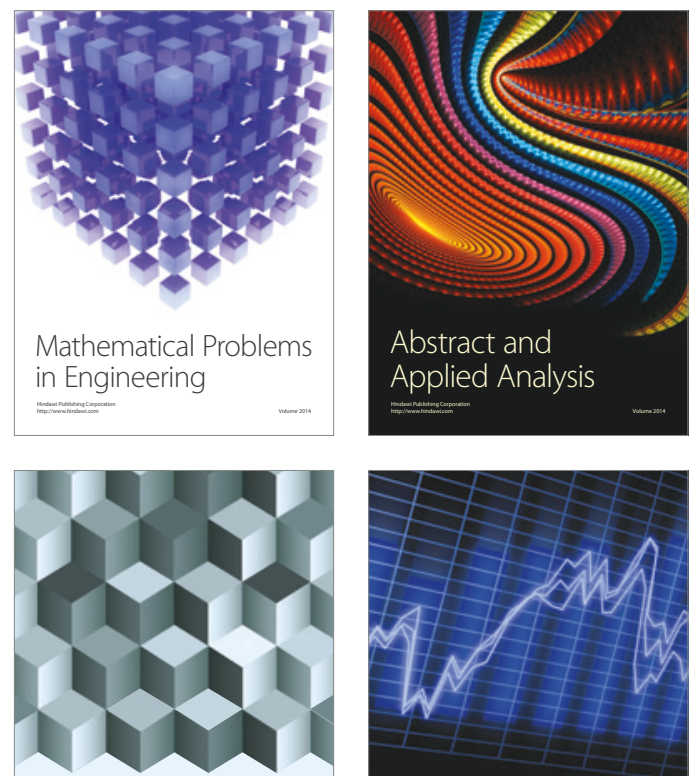

Journal of

Function Spaces

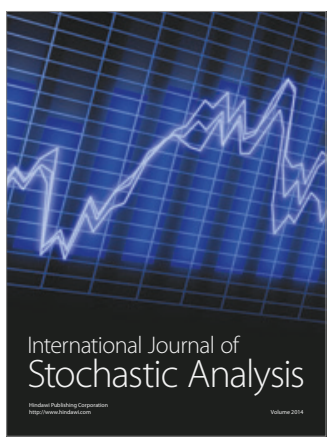

Probability and Statistics
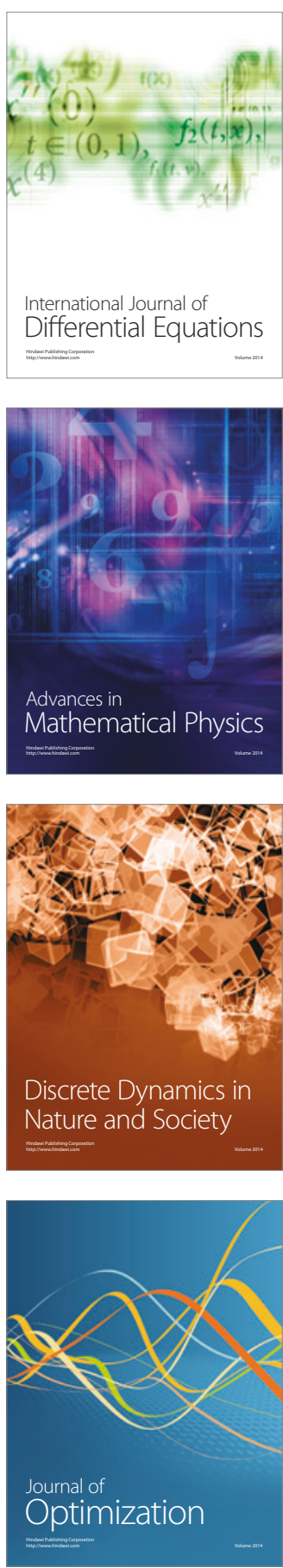